\title{
Analysis of the performance of tapered semiconductor optical amplifiers: role of the taper angle
}

\author{
J. M.G. Tijero, L. Borruel, M. Vilera and I. Esquivias \\ Universidad Politécnica de Madrid, ETSI Telecomunicación-CEMDATIC, Madrid 28040, Spain
}

\begin{abstract}
The beam properties of tapered semiconductor optical amplifiers emitting at $1.57 \mu \mathrm{m}$ are analyzed by means of simulations with a self-consistent steady state electro-optical and thermal simulator. The results indicate that the self-focusing caused by carrier lensing is delayed to higher currents for devices with taper angle slightly higher than the free diffraction angle.
\end{abstract}

\section{INTRODUCTION}

A variety of applications including lidar, material processing, metrology and frequency doubling require high power, high spectral purity and high beam quality laser sources. Although historically solid-state and fiber lasers have been used for these applications, there is an increasing interest in extending the acknowledged advantages of semiconductor lasers to these applications. The master-oscillator poweramplifier (MOPA) architecture with a tapered semiconductor optical amplifier (SOA) acting as the power amplifier is a well suited architecture for this purpose, and the optimization of the design of the tapered SOA is a key point to improve the performance of these devices.

In this work, we use a self-consistent simulation tool for the analysis of the effect of the taper angle on the performance of a two-section high power $1.57 \mu \mathrm{m}$ tapered SOA and derive qualitative design recommendations based on this analysis.

\section{SIMULATION MODEL AND DEVICE DESCRIPTION}

A previously developed model and simulation tool for multicontact tapered lasers [1-2] was slightly modified in order to consider a single pass amplifier instead of a resonant cavity. In brief, the program solves self-consistently the complete steady state electrical, thermal, and optical equations for a single-pass tapered amplifier, assuming a single frequency. The simulator includes a 3D electrical solver of the Poisson and continuity equations coupled to a 3D thermal solver of the heat-flow equation with the local heat sources provided by the electrical solution.

A multiple quantum well InGaAsP/InP epitaxial structure with nominal lasing wavelength close to $1572 \mathrm{~nm}$ was considered in the simulations. The tapered SOAs consist of two separately driven sections: a $0.7 \mathrm{~mm}$ long and $3.5 \mu \mathrm{m}$ wide index guided Ridge Waveguide (RW) section, and a $2 \mathrm{~mm}$ long tapered gain guided section. The calculated full width at $1 / \mathrm{e}^{2}$ of the optical mode at the RW section is $4.9 \mu \mathrm{m}$, yielding a full free diffraction angle at $1 / \mathrm{e}^{2}$ of $7.1^{\circ}$. In the following we will compare the simulation results for three values of the tapered section full angle, $\alpha_{\text {tap }}=4^{\circ}, 6^{\circ}$ and $8^{\circ}$.

The simulations were performed considering a heat-sink temperature of $25^{\circ} \mathrm{C}$, an input power $\left(\mathrm{P}_{\text {in }}\right)$ of $100 \mathrm{~mW}$ and a RW section current $\mathrm{I}_{\mathrm{RW}}$ of $100 \mathrm{~mA}$.

\section{RESULTS AND DISCUSSION}

Fig. 1 shows the simulated output power $\mathrm{P}_{\text {out }}$ and the electrical-to-optical conversion efficiency $\eta_{\mathrm{e}-\mathrm{o}}$ as a function of the taper current $\mathrm{I}_{\text {Tap }}$ for the three values of the taper angle. In each characteristic, the point at the maximum current corresponds to the maximum taper section current with numerical convergence; at a higher current the simulation enters a non-convergent loop which finally produces extremely high internal temperatures. The trend of $\mathrm{P}_{\text {out }}$ and $\eta_{\mathrm{e}-\mathrm{o}}$ with $\alpha_{\text {tap }}$ can be understood by considering the different areas, and therefore, current densities of the devices. At a constant $\mathrm{I}_{\mathrm{TAP}}$, the output power and the efficiency increase when decreasing the taper angle. In consequence, for low and intermediate powers a reference power may be attained at a lower injection with a low angle SOA. However, higher powers are attainable with wider angle devices, at the expenses of a high current through the tapered section.

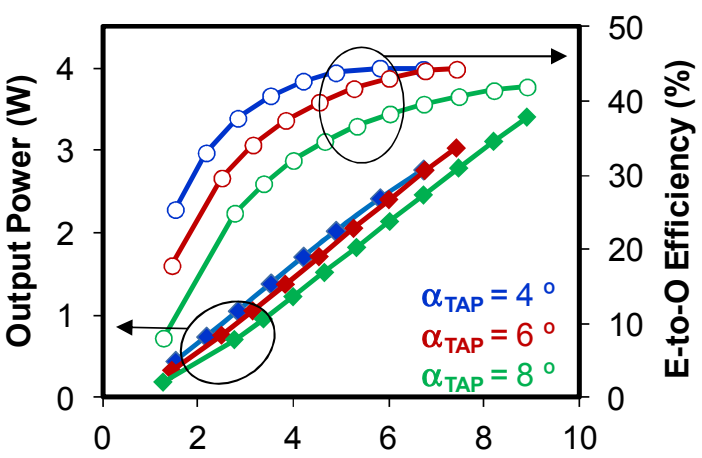

Taper Current (A)

Fig. 1: Simulated $P_{\text {out }}$ and $\eta_{\mathrm{e}-\mathrm{o}}$ as a function of the taper current $\mathrm{I}_{\mathrm{Tap}}$ for the different taper angles.

The evolution of the beam propagation parameter $\left(\mathrm{M}^{2}\right)$ and the beam astigmatism as a function of $\mathrm{I}_{\text {Tap }}$ are shown in Fig. 2 . The increase of $\mathrm{M}^{2}$ and the astigmatism for increasing injected current, commonly observed in tapered lasers [3], are also evidenced here. $\mathrm{M}^{2}$ keeps a relatively low value up to a high current in the case of the $8^{\circ} \mathrm{SOA}$, while the typical abrupt increase of $\mathrm{M}^{2}$ when the beam degrades takes place at lower 
currents in the $6^{\circ}$ and $4^{\circ}$ devices. The evolution of the astigmatism is also in line with this behaviour. The fast increase of astigmatism in the case of the $4^{\circ}$ SOA and the sudden drop to negative values (the simulator does not calculate these values but set them as zero) are clearly indicative of a self-focusing effect which is delayed to a much higher current in the case of the $8^{\circ} \mathrm{SOA}$.

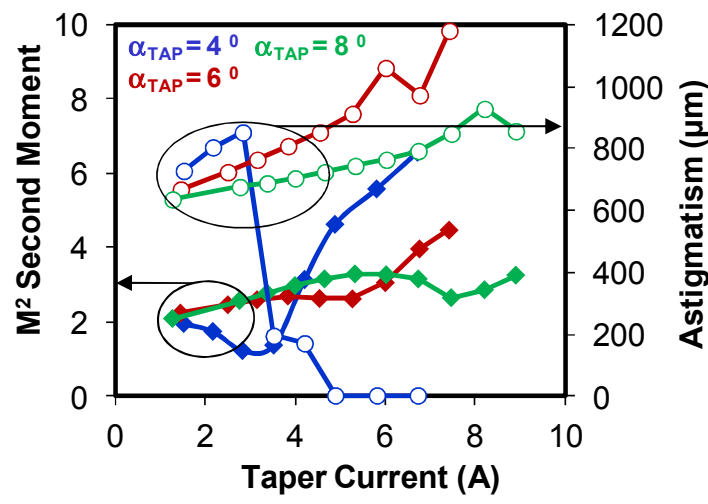

Fig. 2: Simulated beam propagation parameter $\left(\mathrm{M}^{2}\right)$ and beam astigmatism as a function of $\mathrm{I}_{\text {Tap }}$ for the different taper angles.

The self-focusing effect can be clearly observed in Fig. 3, where the near field (NF) at the output facet of the $4^{\circ}$ SOA has been plotted for different $\mathrm{I}_{\text {Tap }}$. At a low current level, the NF shows an almost Gaussian profile, covering most of the output aperture $(\sim 140 \mu \mathrm{m})$. When increasing the current, the shape of the NF evolves to a triangular profile, with a clear narrowing in the center, and finally, at the highest current, the beam selffocuses in an extremely narrow region. This self-focusing, similar to that experimentally observed in $1.5 \mu \mathrm{m}$ tapered lasers [5], is in the origin of the absence of convergence of the simulation code at higher currents.

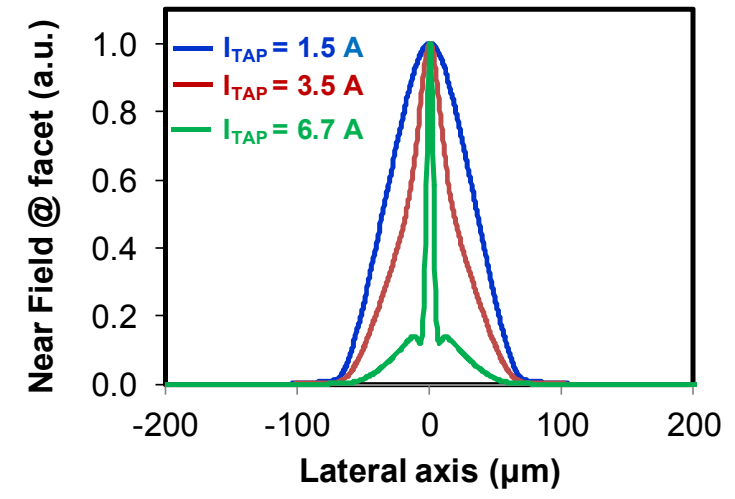

Fig. 3: Near field at facet for the $4^{\circ}$ SOA under different taper section currents.

The beam properties can be better understood with the help of the color maps plotted in Fig. 4. Each map represents, for the $4^{\circ} \mathrm{SOA}$, the photon density in the quantum well plane, normalized for each value of the $\mathrm{z}$ coordinate (longitudinal axis). At a low injection level, the beam leaving the RW section expands and fully covers the tapered section since the taper angle is lower than the free diffraction angle. When increasing the current, the increase of the internal photon density depletes of carriers the central region, giving rise to an increase of the refractive index (carrier lensing effect) which ultimately leads to the self-focusing of the beam.

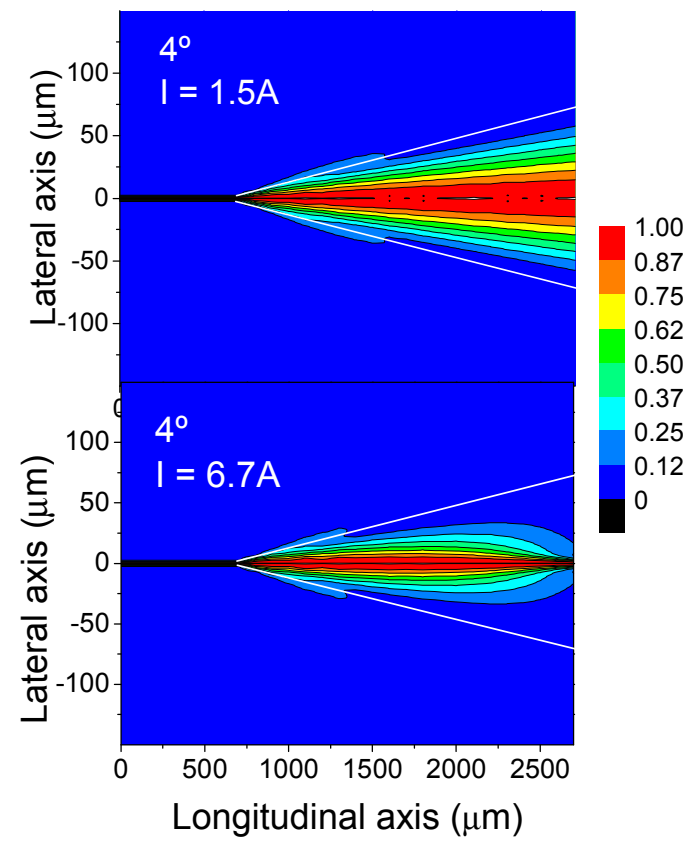

Fig. 4: Color maps of the photon density of the $4^{\circ}$ SOA under low injection (top) and high injection conditions (bottom). The white lines show the borders of the injected region

For wider angle SOAs, the self-focusing effect is delayed to higher output powers, due to the reduction of the internal photon density at equivalent power levels. Therefore, the beam quality improves and higher output powers are attainable.

\section{CONCLUSIONS}

We have applied a self-consistent model to the simulation of $1.57 \mu \mathrm{m}$ tapered amplifiers in order to investigate the relation between beam properties and device geometry. We have found that higher output powers with satisfactory beam quality are attainable with devices with wider taper angle at the expenses of a higher current injected in the tapered section.

\section{ACKNOWLEDGMENT}

This work was supported by the European Commission through the project BRITESPACE under grant agreement no. 313200 and by Ministerio de Economía y Competitividad of Spain under project TEC2012-38864-C03-02.

\section{REFERENCES}

[1] Borruel, L., Sujecki, S., Moreno, P., Wykes, J., Krakowski, M., Sumpf, B., Sewell, P., Auzanneau, S.C., Wenzel, H., Rodríguez, D., Benson, T.M., Larkins, E.C., Esquivias, I., "Quasi-3-D Simulation of HighBrightness Tapered Lasers". IEEE J. Quantum Electron. 40, 463-472 (2004).

[2] Sujecki, S., Borruel, L., Wykes, J., Moreno, P., Sumpf, B., Sewell, P., Wenzel, H., Benson, T.M., Erbert, G., Esquivias, I., Larkins, E.C., "Nonlinear Properties of Tapered Laser Cavities". IEEE J. Select. Topics Quantum Electron. 9, 823-834 (2003).

[3] Donnelly, J.P., Walpole, J.N., Groves, S.H., Bailey, R.J., Missaggia, L.J., Napoleone, A., Reeder, R E., Cook, C.C., "High-power $1.5 \mu \mathrm{m}$ taperedgain-region lasers". Proc. of SPIE, vol. 3284, 54-62 (1998). 